\title{
Blockchain for Reliable and Secure Distributed Communication Channel
}

\author{
Jovan Karamačoski, Nataša Paunkoska and Ninoslav Marina \\ University of Information Science and Technology \\ "St. Paul the Apostle" \\ Ohrid, Macedonia
}

\author{
Magdalena Punčeva \\ University of Applied Sciences \\ and Arts of Western Switzerland \\ Neuchâtel, Switzerland
}

\begin{abstract}
Distributed storage systems (DSS) have a potential of storing big amounts of data. Specialized DSS codes were defined with the main idea to enhance the reliability, availability, maintenance and data security in DSS systems. In this paper, we establish a new way of communication, named Distributed Communication Channel (DCC), that uses the coding mechanism that is similar to the one applied in the DSS. DCC represents a communication channel between two or more parties, that exchange information through multiple physical channels. It adapts the DSS concept using the benefits established with respect to the communication reliability and security against intruders and attacks. Moreover, with this concept we offer an increased security using the recent developments used in the Blockchain technology. The blockchain is used as a tool to securely bypass the transformation matrix or the exchange of encryption keys, between the sender and the receiver in the communication channel. The whole system uses the so-called spatial spreading mechanism to transfer the data through independent channels. Therefore, it is more reliable and secure, than the basic systems using the DSS algorithm.

Index Terms-Distributed storage systems (DSS), Distributed communication channel (DCC), Blockchain, Distributed ledger technologies (DLT), reliability, security, availability.
\end{abstract}

\section{INTRODUCTION}

Linear network coding theory is a fairly new subject in the coding area [1]. It provides enhanced services in the communication between two parties. While still being in development, it lacks practical implementation due to the small number of use cases and the existence of similar and well established P2P solutions. It is mainly seen as a good solution for protection of the communication against security attacks (eavesdropping, snooping, replay attacks, intentional data corruption and jamming). The other use case scenarios include data distribution, throughput increase, relaying, and error management.

Unlike the communication systems, where two parties have a two-way interaction, distributed storage systems most often consists of a single party performing a write and read operation. More precisely, the message is recorded to multiple distributed storage nodes, and it stays there stored in a distributed manner until one wants to retrieve the message. These kind of distributed systems are employing special mechanisms to provide reliable and secure services.

The traditional storage systems that are offering a high level of reliability use the simplest model for increasing reliability. That is the simple data replication model, by replicating the data three times. This is equivalent to the repetition codes used in communications. For companies that are storing petabytes of data, this procedure is expensive as the redundancy is $200 \%$. By the use of more sophisticated coding schemes, this replication model can be substituted by a more efficient model for operation and management. Several related schemes are proposed in [2][8]. The DSS models for data storage are using the same complexity for system management, but the expenditures for failure fixes and management are smaller. This useful advantage of the DSS codes is foreseen as an advantage for future communication channels. If the procedure of coding and dividing the data blocks used in DSS is adapted for the communication channels, the created communication system will have great improvements.

The main idea of the DCC systems is to distribute the segments of a given file through different physically separated communication lines in the network, following the DSS coding procedures that will offer a certain level of security and reliability by adding redundant data in the system. By using DSS coding schemes instead of replication technique as is discussed above, the redundancy can be decreased and the reliability increased. We are proposing implementation of DSS coding schemes in a distributed communication system, as a new approach in this field. The proposal is to apply the procedures of data shredding made by the DSS coding matrix in a communication scenario. The node that generates and encodes the data, uses the DSS matrix. After the coding process finishes, the generated blocks of data are sent through several transmission lines. These lines should be non-correlated and spatially distributed, in order to offer better reliability. The receiving node can receive all the blocks generated by the DSS matrix and can reconstruct the original data. The added redundant data will provide increased reliability in situations where some of the data blocks do not reach the receiver.

The DCC communication system can be seen as a special case of a DSS system where the originating node has a massive failure, after which, a full recovery is needed. The recovery of the data on the original node is possible if one knows the encoding/decoding matrix. In the scenario using DCC, there is a need for secure transfer of the encoding matrix and any featured security keys to the recipient. To obtain a secure transfer of the encoding matrix and security keys, we are proposing a publicly 
accessible Blockchain system for data distribution. Security increasing factor is the spatial spreading of the coded message parts. A potential eavesdropper that can capture a particular block, cannot reveal the whole data. The spatial spreading of the data can offer a certain probability that the eavesdropper can not capture all required packages. The main idea assumes, sending the different block of encoded data through independent paths. This scenario is feasible in a wired network (having several independent wired lines with Internet access), however it is even better when used for wireless network scenarios (wireless sensor networks, multi-RAN 4G and 5G scenarios). The basic DCC system will also employ encryption, to additionally increase the security of the communication. The encoded blocks of data sent through different channels can be encoded with different keys. The process of sharing the keys will be handled by the Blockchain.

In this paper we use the following notation:

- Scalars are denoted by normal lowercase and uppercase letters (e.g., $d, n, k, S, L, u_{1}, u_{S}, c_{n, 1}, c_{n, \alpha}, \alpha, \beta, \psi_{n, d}$ ),

- Vectors are denoted by bold lowercase letters (e.g., u, v),

- Files (sets) are denoted by calligraphic uppercase letters (e.g., $\mathcal{F}, \mathcal{U})$,

- Matrices are denoted by sans-serif uppercase letters (e.g., $\left.\mathrm{C}, \mathrm{I}, \mathrm{M}, \Psi, \mathrm{C}_{s}, \mathrm{D}, \mathrm{U}\right)$,

- Cardinality of a set or a vector is denoted by $|\cdot|$ (e.g., $\mathbf{u}, \mathbf{v})$

- A linear code $\mathrm{C}[n, k, d]$ is a code defined by the generator matrix $C$ and has a codeword length $m$, a message length $k$ and a minimum distance between the codewords $d$.

The paper is organized as follows: Section II gives an overview of the DSS code construction and the functions regarding the process of data encoding, Section III explains the DCC system model that follows the DSS concept and how this proposal enables security using the Blockchain method. The DCC procedures, advantages, and disadvantages are elaborated in Section IV and Section V concludes the paper.

\section{OVERVIEW OF DSS CODE CONSTRUCTION AND FUNCTIONING}

The proposed DCC system concept applies the DSS functions, but also corresponds with the Blockchain database. Therefore, in this section, first we descried the functioning and the construction of a DSS system. As a concrete DSS code, we use the product-matrix code.

The Distributed storage system (DSS) is a network constructed by a large number of interconnected nodes, that manage a big amount of data. Potential node failure emphasizes the importance of data reliability. By introducing redundancy in the system, we can protect data integrity from node failures. The easiest form of redundancy is data replication, having multiple copies of data on different nodes, but this is not efficient as introduces a big overhead. The first solution towards solving this problem is using the traditional Reed-Solomon codes and other erasures codes instead of data replication [2], [8]-[11].
In general, the problem can be cast as storing a file $\mathcal{F}$ of $S$ $q$-ary symbols using DSS $[n, k, d]$ code. The file is written as a sequence of $q$-ary symbols $\mathbf{u}=\left\{u_{1} u_{2} \ldots u_{S}\right\}$, where $|\mathbf{u}|$ is the size. This file is stored on $n$ servers (nodes). For simplicity, the size of the sequence $\mathbf{u}$ is an integer multiple of the parameter $S$, namely $|\mathbf{u}|=L S$ and here we will consider the case $L=1$. After the $S$ is segmented in $k$ pieces, vectors of size $\alpha$ is stored in each of $n$ distinct nodes.

In case of node failure or partial data loss, two important data retrieval processes happen in DSS: the reconstruction and the repair. The reconstruction process takes place when the user (data collector) wants to retrieve the information stored in the system. In this case, the data collector contacts any set of $k$ nodes and downloads all $\alpha$ symbols stored on them and by processing them gets the entire message $\mathcal{F}$ of size $S$. The repair process is activated whenever a node in the system, which stores $\alpha$ symbols, fails. A new node that has to replace the node under failure contacts a set of $d(n-1 \geq d \geq k)$ alive nodes in the network and downloads $d \beta$ symbols, where $\beta \leq \alpha$. The downloaded data from the $d$ nodes after that is used to recover the lost data that was stored in the failed node.

Although distributed storage systems have benefited largely from the past research in the area of coding for communication channels, there are several key aspects of a networked storage system that are not addressed in the classical coding theory. Thus, more sophisticated solutions are the codes introduced by Dimakis in [3], [12], called regenerating codes. A lot of authors work in the direction of improving the work of Dimakis. One case, which will be used in our paper, is the specially developed codes known as product-matrix codes. Details and theoretical explanation about these codes can be found in [5].

The DSS construction process is following. The code that is used has parameters $[k, n, d]$. The parameters are $k$ (number of nodes contacted by the data collector for the reconstruction of the entire message), $n$ (number of storage nodes in the distributed system) and $d$ (number of nodes contacted during the repair process, i.e., data recovery of the failed node). The encoding will be done on a file $\mathcal{F}$ of size $|\mathcal{F}|$ bits, equivalent to $S q$-ary symbols represented by the vector $\mathbf{u}=\left(u_{1}, u_{2}, \ldots, u_{S}\right)$. The vector is arranged in the form of a message matrix $M$ consisting of all original symbols plus the redundant ones. In order to store the message in a dispersed and distributed system, it is used another encoding matrix $\Psi$ of dimension $(n \times d)$ that is invertible for regularly performing the reconstruction and the repair processes. Thus the constructed code can be obtained as a multiplication between the encoding matrix and the message matrix

$$
\mathrm{C}=\Psi \cdot \mathrm{M} \text {. }
$$

\section{DCC SYSTEM MODEL}

In a DCC scenario, where the node uses the DSS coding scheme, the encoder will create the code-matrix $C$, where instead of storing the $n$ encoded symbols in $n$ nodes, the sender will send the data through $n$ different paths (physical channels). The encoded symbols should be routed through the 
network to the destination node. In a wired network scenario, the different paths will be different Internet providers (with spatially uncorrelated backbone links in order to increase the reliability of the protocol). The DCC protocol will be of bigger importance for wireless scenarios (especially wireless sensor networks with a high amount of distributed nodes). In wireless sensor networks, the DCC protocol will leverage the security of the communication. This is of a great importance in a scenarios related to defense and military. The protocol determines $n$ spatially uncorrelated paths, to deliver the data. The DCC encoding procedure will protect the information from eavesdroppers, and from partial data loss.

Both sides of the communication channel can pre-agree an encoding (decoding) matrix via the off-line method. Knowing the disadvantages of off-line secrecy sharing, and the problem of key (and encoding matrix) refresh (renewal), the DCC protocol offers a Blockchain based solution for Public Key and Encoding Matrix Infrastructure, shown in Fig. 1. The DCC Blockchain part will employ publicly available Blockchain interface for key and encoding matrix exchange. The online key and encoding matrix exchange have several advantages over the off-line key exchange. The implementation of this mechanism by the use of Blockchain, will create a server-less system, robust and durable, with easy access control mechanisms. This way the access to the sent data can be given or restricted through the Blockchain interface.

The procedure for establishing a communication channel between the sender and the receiver is as follows:

1) Sender encodes the file and generates the code matrix $C$.

2) Sender sends the encoding matrix $\Psi$ to the Blockchain PKI-like system (optionally initiates a key exchange procedure). PKI stands for public-key infrastructure.

3) Sender selects $n$ distinct paths (different upstream providers or different routes).

4) Sender sends the blocks through the distributed channels.

5) Receiver receives the blocks.

6) Receiver looks up into the Blockchain public database of the encoding matrix $\Psi$ and generates the decoding matrix (optionally stores the decryption code).

7) Receiver decodes the message.

The security of the communication between the nodes primarily relays on the information-theoretic secrecy, obtained by the DSS code in the DSS environment. Some research regarding this type of secrecy in DSS is given in [13]-[16]. DCC concept can also count on the information-theoretic secrecy due to its distributed nature and plus of the security that will offer the Blockchain database. That is not enough, if we want to guarantee really strong security. Depending on the required level of security, system complexity and communication node capabilities, the system can employ additionally encryption mechanisms. The encryption in the system can be implemented in the pre-encoding stage and/or post-encoding stage. In the preencoding stage, the message $M$ will be encoded beforehand and after that, the codeword symbols sent through different paths.
The post-encoding stage will take place at the moment before the codeword symbols are sent through different paths. In the scenario described above, the protocol can have two approaches: one is to use the same key for encryption for every $n$ packages sent through $n$ separate paths, and the other is using $n$ different encryption keys for $n$ separate paths.

\section{A. Blockchain as a matrix exchange infrastructure}

The encoding matrix $\Psi$, used in the DSS coding procedure is chosen from a set of optimal coding matrices, previously determined with the procedure explained in [5]. For this coding scheme to work, both sides have to use the same coding matrix. Both sides can select a coding matrix and agree for the matrix by using an on-line or off-line method. The off-line methods are basically impractical because they are complex and the transfer of the agreed coding matrix is non-trivial. Opposite to this method, the online method offers flawless integration of the agreed matrix to the system. Basically, the online method will employ direct peer-to-peer agreement between the parties through e-mails, or by picking one from the pre-calculated set stored in a server or by use of the most modern approach build on Blockchain. The Blockchain is the most recent technology that offers a secure, private, and reliable way for an on-line agreement between the parties.

The Blockchain is a type of dynamic database capable to store data of any type. The access control to the data is managed by the use of Smart Contracts. The Blockchain database will be entity accessible by everyone who has a connection to the public Internet. The parties securely will agree for one coding matrix and use that matrix for the whole communication session, or the coding matrix can be changed per message. Because of the distributed nature of the Blockchain network, access to the data cannot be restricted. Also, the data in the Blockchain is reusable, so any recall for information is always possible. The primary protection provided with the Blockchain is privacy. The integration of Tor [17] as part of the Blockchain database can further increase the privacy and security of the communication.

\section{B. General DCC construction concept}

The DCC model implements Blockchain-based database to store the $\Psi$ matrices (see Fig. 1). One can use the Blockchain database to reference an existing unencrypted $\Psi$ matrix or to upload an encrypted version of a particular $\Psi$ matrix and reference it anytime when he wants to share the information with other parties in the communication system. The other segment of the system, the DCC communication channel, is used to compute the secret data between the parties.

In the DCC scenario, we always have two sides for the communication, the sender and the receiver. In this case, the message between both parties is exchanged following the DSS distribution model. The DSS code uses the $\mathrm{C}[n, k, d]$ code for message encoding and distribution. In DCC the same parameters are considered in order to perform encoding and delivery of the message, so we can denote the DCC code as $\mathrm{C}[n, k, d]$. 


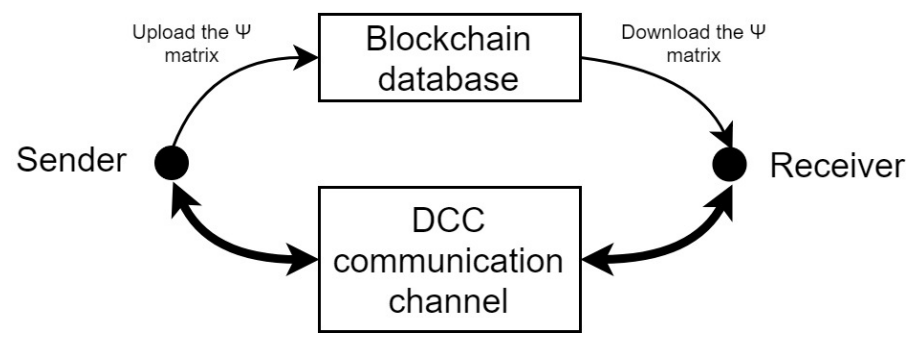

Fig. 1. DCC system model.

The file that needs to be sent, has size $|\mathcal{F}|$. Firstly, at the sender side, it is split into smaller pieces of size $S$ and then each piece is encoded using the $\mathrm{C}$ code. Each message of $S$ $q$-ary symbols is represented by the vector

$$
\mathbf{u}=\left(u_{1}, u_{2}, \ldots, u_{S}\right)
$$

Following the DSS code construction, given in [5], we choose $S$ to be $S=k \alpha$. Hence, the size of the block that going to be sent through different providers (channels) will be $\alpha=d-k+1$, where $d=n-1$.

After setting up all parameters, next step is arranging the message matrix $\mathrm{M}$ of size $(d \times \alpha)$ by filling up all positions in it with the message symbols from the set $\left\{u_{i}\right\}_{i=1}^{S}$. The rule for how to arrange the matrix is following, the upper part of the matrix or the submatrix of dimension $(\alpha \times \alpha)$ need to be symmetric. Same for the lower part of the matrix of dimension $(\alpha \times \alpha)$, again need to be symmetric. The general message matrix is given as

$$
\mathbf{M}=\left(\begin{array}{cccc}
m_{1,1} & m_{1,2} & \ldots & m_{1, \alpha} \\
m_{2,1} & m_{2,2} & \ldots & m_{2, \alpha} \\
\vdots & \vdots & \ddots & \vdots \\
m_{d, 1} & m_{d, 2} & \ldots & m_{d, \alpha}
\end{array}\right)
$$

The encoding matrix $\Psi$ with dimension $(n \times d)$, which is crucial in the Blockchain part for the safety reason, has the following shape

$$
\boldsymbol{\Psi}=\left(\begin{array}{cccc}
\psi_{1,1} & \psi_{1,2} & \ldots & \psi_{1, d} \\
\psi_{2,1} & \psi_{2,2} & \ldots & \psi_{2, d} \\
\vdots & \vdots & \ddots & \vdots \\
\psi_{n, 1} & \psi_{n, 2} & \ldots & \psi_{n, d}
\end{array}\right) .
$$

The matrix $\Psi$ has to be a so-called Cauchy matrix since such matrices are applicable in our proposed DCC scheme and in an effective way can perform the reconstruction process to obtain the original message. Cauchy matrix is a matrix with special construction such that any element of the matrix $\psi_{i, j}$, has the following property $\psi_{i, j}=1 /\left(x_{i}-y_{j}\right)$, such that $x_{i} \neq y_{j}$, for all $i=1, \ldots, n$, and all $j=1, \ldots, d$. It has the property that any submatrix is again a Cauchy matrix. Additionally, any square Cauchy matrix and submatrix is invertible, which efficiently performs the decoding process [5], [16]. This matrix is sent to the Blockchain database.
Thus the DCC constructed code can be obtained as a multiplication between the encoding (generator) matrix and the message matrix

$$
\mathrm{C}=\Psi \cdot \mathrm{M} \text {. }
$$

The final result will give the DCC code matrix

$$
\mathrm{C}=\left(\begin{array}{cccc}
c_{1,1} & c_{1,2} & \ldots & c_{1, \alpha} \\
c_{2,1} & c_{2,2} & \ldots & c_{2, \alpha} \\
\vdots & \vdots & \ddots & \vdots \\
c_{n, 1} & c_{n, 2} & \ldots & c_{n, \alpha}
\end{array}\right) .
$$

Then, the sender took row by row $\left\{\left[c_{1,1} c_{1,2} \ldots c_{i, \alpha}\right]\right\}_{i=1}^{n}$ from the DCC code matrix, choose $n$ distinct paths and send the blocks (one block represent one row form the matrix) through the different routes to the receiver side. All blocks are indexed with a cardinal number of the DCC code matrix row. From coding theory we know that the decoding matrix $\Psi_{d}$ can be easily computed from the encoding (generator) matrix $\Psi$. To explain briefly that, we remind that from any encoding (generator) matrix, we can obtain the corresponding standard form of the generator matrix $\mathrm{G}$ in a form $\mathrm{G}=\left[\mathrm{P}^{T} \mid \mathrm{I}_{d}\right]^{T}$. Matrix $\mathrm{G}$ is obtained by re-arranging the columns and rows of the generator matrix $\psi$ such that it gets the for that in the lower part of the matrix we get the identity matrix $\mathrm{I}_{d}$ of dimension $d$. Then in the upper part we get the matrix $\mathrm{P}$ of size $(n-d) \times d$. When the standard generator matrix is used, we get the socalled systematic code $\mathrm{C}_{1}=\mathrm{G}$. M. In the systematic code, the first symbols represent the message symbols while the last symbols are called parity check symbols. As we know from coding theory, the parity check symbols are used to correct the errors in the message and that is the main purpose of the error-correcting linear codes. From algebra, it is also known that the standard matrix $G$ can be obtained from any non-standard generator matrix $\Psi$ as $U \Psi=G$, where $U$ is a unique invertible matrix. Codes obtained from generator matrices related in this manner are called equivalent codes. In our case, since we multiply the message matrix by the generator matrix from left, the first $(n-d) \alpha$ symbols are the parity check symbols. They are placed in the upper $(n-d) \times \alpha$ sub-matrix of the matrix $C_{s}$. The message itself will be in the last $d \alpha$ symbols, represented by the $d \times \alpha$ sub-matrix of the matrix $\mathrm{C}_{s}$. Our systematic code is

$$
\mathrm{C}_{s}=\left(\begin{array}{c}
\mathrm{PM} \\
\mathrm{M}
\end{array}\right)
$$

So we see that the original message $\mathrm{M}$ is in the lower $(d \times \alpha)$ submatrix of the matrix $C_{s}$ and can be easily obtained by cutting that part or multiplying by the $(d \times n)$ matrix $\mathrm{D}=\left[\mathrm{O} \mid \mathrm{I}_{d}\right]$, where $\mathrm{O}$ is the all-zero matrix of size $d \times(n-d)$. Then we have $\mathrm{C}_{s}=\mathrm{GM}=\mathrm{U} \Psi \mathrm{M}=\mathrm{UC}$. From this little reminder, we get that the message is reconstructed as $\mathrm{M}=\mathrm{DC}_{s}=\mathrm{DUC}$.

\section{Reconstruction in DCC}

In order to reconstruct the original message, the receiver receives all separate blocks, and by using the indices can construct the DCC code matrix $\tilde{C}$. Next thing is the communication 
between the receiver and the Blockchain database, for obtaining the encoding matrix $\Psi$ needed for decoding. Now the end user is ready to decode and see the content of the message, by multiplying the decoding matrix and the received DCC coding matrix as

$$
\mathrm{M}=\Psi_{d} \cdot \tilde{\mathrm{C}}
$$

where $\Psi_{d}$ is the decoding matrix that can easily be obtained from $\Psi$. This is possible since $\psi$ and any of its submatrices are Cachy matrices. In the sequel, we explain that.

In the DSS scenario, the reconstruction process allows the original message to be obtained using data stored on $k$ nodes instead using data from all $n$ nodes $(n>k)$. Thus, the receiver is able to decode the message using only $k$ out of $n$ symbols, i.e., different paths. This possibility increases the communication system reliability and security against the intruders. The reliability is upgraded in a sense of communication errors or fails of some of the used transmission paths. The security is increased by means of intruder inability to approach all different $k$ provider paths and catch the blocks from them.

After the receiver contacts the Blockchain database to get the encoding matrix, he can decode the message. Hence, if there are some transmission paths failures or knowledge that there is some intruder attack, the receiver can use the corresponding $k \times d$ submatrix from the encoding matrix and $k$ blocks to recover the original message. The process is the following: the encoding submatrix $\tilde{\Psi}$ of dimension $(k \times d)$ has $k$ rows, depending exactly on the received indices of the blocks, i.e., which blocks are going to be used in the decoding process. The encoding submatrix now has the following shape

$$
\tilde{\Psi}=\left(\begin{array}{cccc}
\psi_{i_{1}, 1} & \psi_{i_{1}, 2} & \ldots & \psi_{i_{1}, d} \\
\psi_{i_{2}, 1} & \psi_{i_{2}, 2} & \ldots & \psi_{i_{2}, d} \\
\vdots & \vdots & \ddots & \vdots \\
\psi_{i_{k}, 1} & \psi_{i_{k}, 2} & \ldots & \psi_{i_{k}, d}
\end{array}\right)
$$

The original message then can be obtained similarly as explained above, by multiplying

$$
\mathrm{M}=\tilde{D} \tilde{U} \tilde{C}
$$

where $\tilde{C}$ represents the DCC coding matrix consist of $k$ different received blocks from $k$ providers. Here again it is easy to calculate $\tilde{U}$ as the matrix to obtain the standard generator matrix $\tilde{\mathrm{G}}=\tilde{U} \tilde{\Psi}$ of size $k \times d$. Similarly as before, here $\tilde{U}$ is an invertible $k \times k$ matrix that converts the matrix $\tilde{\Psi}$ of size $k \times d$ into the matrix $\tilde{G}$ of the same size. Then it is easy to see that the decoding (reconstruction) matrix is $\tilde{\Psi}_{d}=\tilde{D} \tilde{U}$, and can be easily recovered from $\Psi$ and the indices of the received paths, which correspond to the indices of the $k$ out of $n$ rows in matrix $\Psi$.

\section{Concrete DCC construction concept}

Lets have a DCC code given by $\mathrm{C}[4,2,3]$. The message size that can be sent through the DCC system in one sequence is $S=k \alpha=2 \cdot 2=4$, where $\alpha=d-k+1=2$. The vector representing the message is $\mathbf{u}=\left(u_{1}, u_{2}, u_{3}, u_{4}\right)$.
This vector need to be arranged in a message matrix $M$ of size $(d \times \alpha)=(3 \times 2)$. Following the structure of the message matrix explained in III-B, it obtains the shape

$$
\mathbf{M}=\left(\begin{array}{ll}
u_{1} & u_{2} \\
u_{2} & u_{3} \\
u_{3} & u_{4}
\end{array}\right)
$$

The encoding matrix $\Psi$ of dimension $(n \times d)=(4 \times 3)$ that will be used in the construction, is sent to the Blockchain database, where can securely be picked up by the receiver. In this example, the matrix looks likes

$$
\Psi=\left(\begin{array}{lll}
1 & 0 & 0 \\
0 & 0 & 1 \\
1 & 1 & 1 \\
1 & 2 & 3
\end{array}\right)
$$

The DCC construction code done by multiplying the encoding and message matrices will be

$$
\begin{aligned}
\mathbf{C} & =\left(\begin{array}{cc}
c_{1,1} & c_{1,2} \\
c_{2,1} & c_{2,2} \\
c_{3,1} & c_{3,2} \\
c_{4,1} & c_{4,2}
\end{array}\right) \\
& =\left(\begin{array}{cc}
u_{1} & u_{2} \\
u_{3} & u_{4} \\
u_{1}+u_{2}+u_{3} & u_{2}+u_{3}+u_{4} \\
u_{1}+2 u_{2}+3 u_{3} & u_{2}+2 u_{3}+3 u_{4}
\end{array}\right) .
\end{aligned}
$$

The sender now has four blocks (codewords), represented by each row of the $C$ and it needs four different paths (providers) to send them to the receiver side. The receiver now needs to contact the Blockchain database and take from there the encoding matrix. After that, the decoding process is just multiplying of the received blocks in the form of a matrix with the inverse encoding matrix.

If there is a situation of transmission path failures or intruders attack, in other words, the security is jeopardized, the receiver is able to bypass the malicious blocks and used the rest $k$ blocks to recover the original message.

If the 2nd and 3rd blocks are lost the received DCC code matrix will be

$$
\begin{aligned}
\tilde{\mathrm{C}}_{\left(i_{1}, i_{4}\right)} & =\left(\begin{array}{cc}
c_{1,1} & c_{1,2} \\
c_{4,1} & c_{4,2}
\end{array}\right) \\
& =\left(\begin{array}{cc}
u_{1} & u_{2} \\
u_{1}+2 u_{2}+3 u_{3} & u_{2}+2 u_{3}+3 u_{4}
\end{array}\right) .
\end{aligned}
$$

Thus the original message can be recovered as explained above, namely

$$
M=\tilde{\Psi} \tilde{C}
$$

It is also obvious that we have four linear equations with four variables $\left(u_{1}, u_{2}, u_{3}, u_{4}\right)$ and we can reconstruct the original message (matrix) $\mathrm{M}$. 


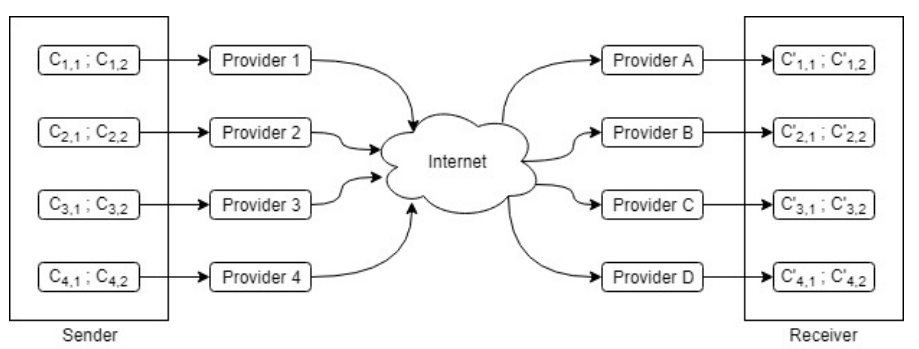

Fig. 2. The basic DCC scenario.

\section{DCC PROCEDURE}

The DCC procedure is a type of network coding where the two parties of the communication channel are securing the transmission line between them and are offering a high level of reliability in the communication process. The procedure will employ a higher number of physical transmission lines to ensure a high level of non-correlation of the paths.

To explain the procedure we are taking into account an example with 4 physical transmission lines or 4 Internet providers. The scenario is depicted in Fig. 2. In this figure, we are not showing the DSS part and the coding procedure in order to simplify the diagram (this part is explained in Section II). The coding procedure, through example, is explained in the previous section. The figure shows the physical part of the communication link between the two transceivers. For most beneficial performances, the encoded message, or the codeword should have the same number of symbols, as the number of physical Internet lines. According to the figure, we are showing an example of a coding scheme with $n=4$ symbol codewords.

This procedure offers several benefits, but the two main benefits are:

(1) Improving the reliability, i.e., the probability that the information is delivered without errors, and

(2) Protecting the transmission line from eavesdropping by shredding the data and transmitting the pieces through different paths.

Continuing with the main scenario, and using the four symbol codewords example, in Fig. 3, we can visualize the potential of the DCC procedure. Note that in this example, the number of message symbols is $k=2$. The figure shows the ability of the DCC procedure to obtain errorless communication even in the presence of severe channel failures. This means that in the worst scenario, where we have $k=2, n=4$ and we have $f=2$ failures, we can still recover the whole message at the receiver side.

The second benefit, the protection from an eavesdropper is shown in Fig. 4. Knowing the DSS capabilities and limitations, in order to protect the message from reconstruction by eavesdroppers, the number of captured symbol codewords should be lower than $n-k-1$. According to this restriction, our example shows a capability to protect the message if less than two codeword symbols are captured (in this case only one).

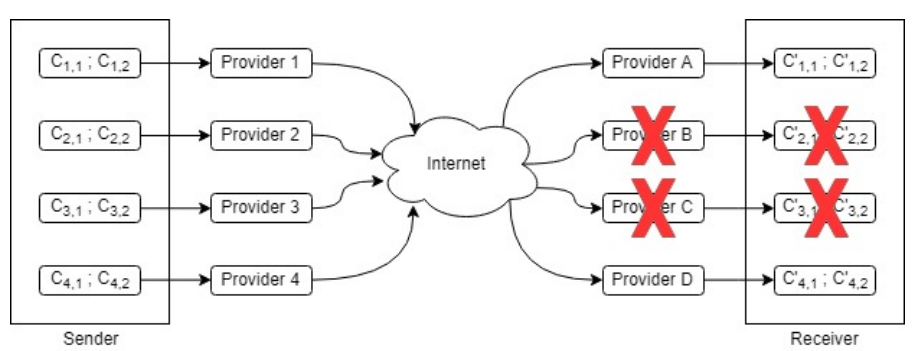

Fig. 3. DCC scenario of link failure protection.

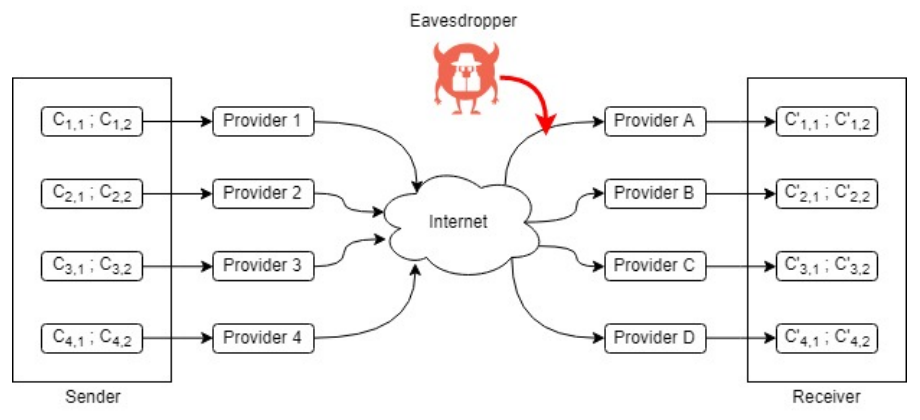

Fig. 4. DCC scenario of eavesdropping protection.

The DSS codes have a weakness. To set the whole picture we are mentioning the worst-case scenario. If the eavesdropper can access the content of more than $n-k-1$ codeword symbols or at least two data stream according to our example, as it is shown in Fig. 5, this means that he can reconstruct the data and the information theoretic secrecy fails. This scenario is pretty rare to occur, but to be on the safe side, our proposal is to implement encryption layer, either before the encoding phase to take place (pre-encoding encryption), or after the encoding phase to take place (post-encoding encryption). The schematic for the pre-encoding encryption is presented in Fig. 6 and the schematic for post-encoding encryption is presented in Fig 7. The decision which approach will take place depends on the level of security one wants to obtain, the processing power of the equipment and the complexity of the system.

The DCC system has a number of advantages regarding the increased security and reliability of the communication. Besides

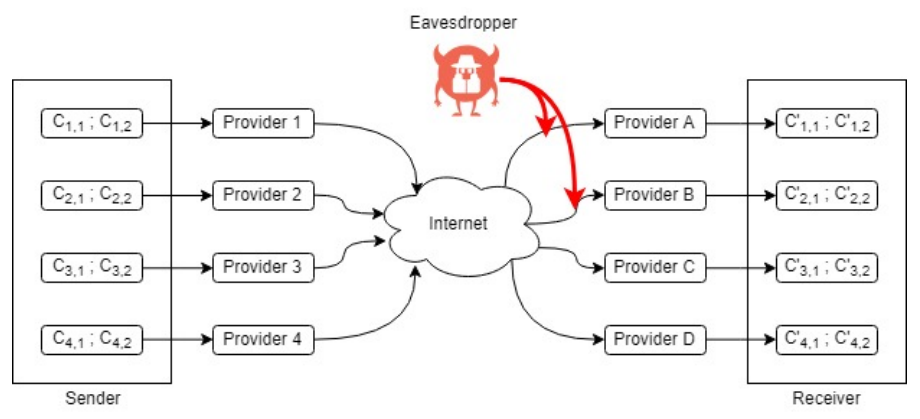

Fig. 5. DCC scenario of failure to protect from eavesdropping. 


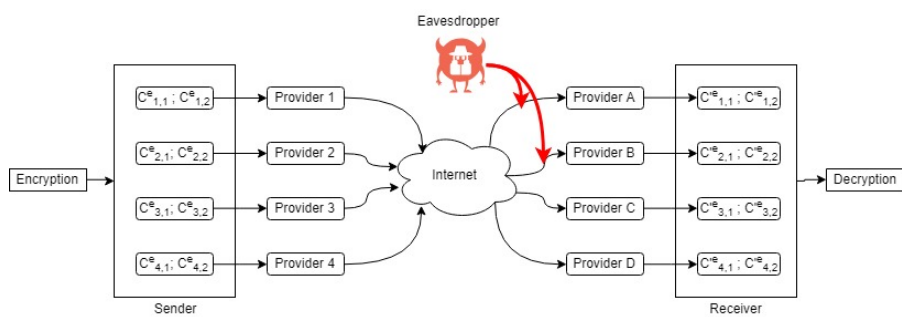

Fig. 6. DCC scenario of pre-encoding encryption protection.

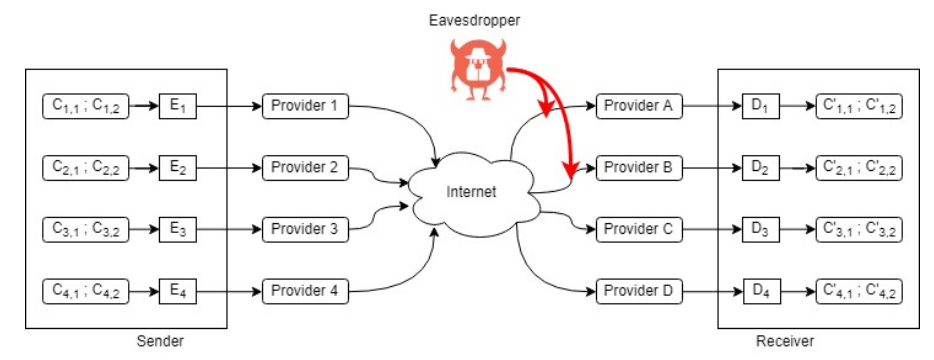

Fig. 7. DCC scenario of post-encoding encryption protection.

the advantages, this system has a disadvantage regarding the increased complexity of the system. The need for a high level of uncorrelated physical paths in a wired network scenario is a problem, due to a small number of potential Internet providers. In a general wireless network scenario, the provision of several uncorrelated physical paths can be mitigated in an easier manner. The most suitable implementation scenario for a DCC system will be in a dense wireless sensor networks or next generation relay networks, or scenarios of military networks and postdisaster ad-hoc networks.

\section{CONCLUSION}

The advance of the Blockchain technology and the increased implementation it in different use-case scenarios, lead us to the idea of implementing the Blockchain distributed database for the purposes of a DCC communication system. The benefits of the Blockchain technology, like the increased security and privacy, the distributed fashion of the database and the ability of the Blockchain to be automated, will increase the security of the DCC communication system. The system will provide a multidimensional increase in security of a communication channel with a slight increase in the complexity of the system as a whole. The system incorporates two levels of security. One level of security is provided by the information-theoretic secrecy provided by the DSS encoding process and the other level is the implemented encryption in the pre-encoding or post-encoding stage. The Blockchain technology will complement to these two levels of security by providing a safe public database for encoding matrix and encryption key exchange.

\section{REFERENCES}

[1] R. W. Yeung, Information theory and network coding. Springer Science \& Business Media, 2008.

[2] H. Weatherspoon and J. Kubiatowicz, "Erasure coding vs. replication: A quantitative comparison," in Proc. 1st Int. Workshop Peer-to-Peer Syst. (IPTPS), pp. 328-338, 2001.

[3] A. G. Dimakis, P. B. Godfrey, Y. Wu, and K. Ramchandran, "Network coding for distributed storage systems," IEEE Trans. on Inf. Theory, vol. 56, no. 9 , pp. 4539-4551, Sep. 2010.

[4] K. V. Rashmi, N. B. Shah, and P. V. Kumar, "Regenerating codes for errors and erasures in distributed storage," in Proc. IEEE Int. Symp. on Inf. Theory (ISIT), Jul. 2012.

[5] K. V. Rashmi, N. B. Shah, and P. V. Kumar, "Optimal exact-regenerating codes for distributed storage at the MSR and MBR points via a productmatrix construction," vol. 57, no. 8, pp. 5227-5239, Aug 2011.

[6] A. S. Rawat, O. O. Koyluoglu, N. Silberstein, and S. Vishwanath, "Optimal locally repairable and secure codes for distributed storage systems," IEEE Trans. on Inf. Theory, vol. 60, pp. 212-236, Nov. 2013.

[7] Y. Wu, A. G. Dimakis, and K. Ramchandran, "Deterministic regenerating codes for distributed storage," in The Allerton Con. Control, Computing, and Communication, Sep. 2007.

[8] A. G. Dimakis, V. Prabhakaran, and K. Ramchandran, "Decentralized erasure codes for distributed networked storage," IEEE Trans. on Inf. Theory, vol. 52, no. 6, pp. 2809 - 2816, Jun. 2006.

[9] M. Sathiamoorthy, M. Asteris, D. Papailiopoulos, A. G. Dimakis, R. Vadali, S. Chen, and D. Borthakur, "Xoring elephants: Novel erasure codes for big data," in In Proceedings of the VLDB Endowment, vol. 6, pp. 325-336, 2013.

[10] V. Guruswami and t. . M. Wootters, "Repairing Reed-Solomon Codes," in Trans. Inf. theory IEEE, vol. 63, pp. 5684 - 5698, Sep. 2017.

[11] A.S. Rawat, I. Tamo, V. Guruswami and K. Efremenko, "MDS code constructions with small sub-packetization and near-optimal repair bandwidth," in Trans. Inf. Theory IEEE, vol. 64, pp. 6506-6525, Oct. 2018.

[12] A. G. Dimakis, P. B. Godfrey, M. Wainright, and K. Ramchadran, "Network coding for distributed storage systems," in Proc 26th IEEE Int. Conf. Computer Communications, pp. 2000-2008, May 2007.

[13] N. B. Shah, K. V. Rashmi, and P. V. Kumar, "Information-theoretically secure regenerating codes for distributed storage," in Proc. of IEEE Globecom, Mar. 2011.

[14] S. Pawar and S. El Rouayheb and K. Ramchandran, "On secure distributed data storage under repair dynamics," in Proc. Int. Symp. on Inf. Theory, Jun. 2010.

[15] S. Goparaju, S. El Rouayheb, R. Calderbank, and H. V. Poor, "Data secrecy in distributed storage systems under exact repair," NetCod, pp. 1-6, 2013.

[16] N. Paunkoska, N. Marina, W. Finamore, and J. Karamachoski, "Secure shortened MSR codes," in International Wireless Communications and Mobile Computing Conference (IWCMC), Jun. 2017.

[17] P. Syverson, R. Dingledine, and N. Mathewson, "Tor: The secondgeneration onion router," in Usenix Security, 2004. 